\title{
A Preview of the NIST/TMS Additive Manufacturing Benchmark Test and Conference Series
}

\author{
Lyle Levine, Mark Stoudt, Brandon Lane
}

Additive manufacturing (AM) is widely recognized as a transformative technology that facilitates production of threedimensional metal and polymeric parts with intricate geometries that often prove too costly, difficult, or in some cases, impossible to produce using traditional subtractive manufacturing processes. However, the extreme thermal conditions generated during the AM process often create microstructures that have steep compositional gradients and unexpected phases. The accepted post-process heat treatments, which are based on nominal compositions and equilibrium phase diagrams, are ineffective for many AM materials. More importantly, application of these heat treatments could produce parts with severely degraded performance and unexpectedly short service life.

The combination of a rapidly changing technology and a lack of material property data has exacerbated the need for traceable standards and benchmark tests.

In response to this need, the framework to develop a set of benchmark tests specifically designed to meet the needs of the additive manufacturing community was proposed at an AM workshop at the National Academies in Washington, D.C in October of 2015. Within weeks, the AM community responded as researchers from dozens of organizations around the world volunteered their time and input to make the Additive Manufacturing Benchmark (AM-Bench) test series a reality. Today, the AM-Bench committees include over 70 scientists and engineers from more than 50 organizations, including the National Institute of Standards and Technology
(NIST), five Department of Energy laboratories, six NASA centers, three Department of Defense laboratories, and numerous companies, universities, and other research centers in North America, Europe, and Asia.

Why was developing a set of benchmark tests so well received? Just like the technology it represents, the answer to this question is multifaceted and complex. Unlike most traditional manufacturing methods, AM combines material processing and shaping into a single step and the material structure and properties can vary considerably, depending upon the local build conditions. Characterizing the variations in microstructure is an expensive and time-consuming process, so a considerable gap has developed between improvements in the process technology and material quality. For this reason, computer simulation has become the essential tool required to bridge this technological gap and refine the process so it can reproducibly create high-value parts with both the previously mentioned intricate geometries and reliable performance. The challenge is integrating and validating the variety of computer simulations and models over the needed time and length-scales for AM. Accomplishing this requires both validated data and specific metrics to gauge the accuracy - and AM-Bench is designed to provide both.

Modeling software is being developed for part design, process modeling, process mapping, defect avoidance, residual stresses, part distortion,

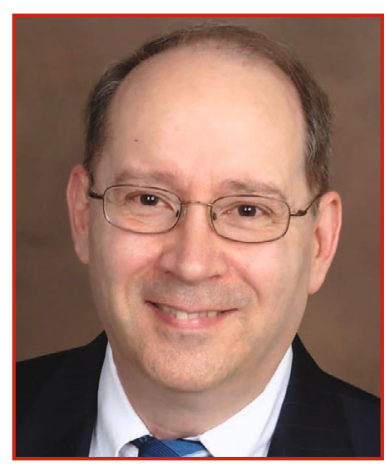

Lyle Levine

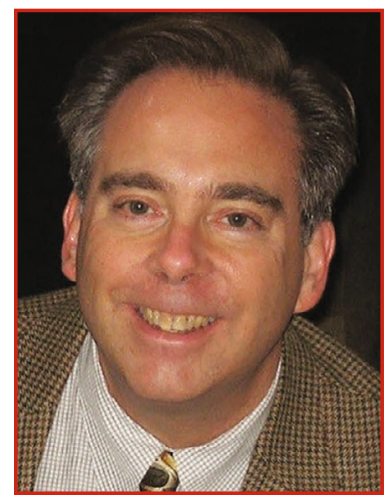

Mark Stoudt

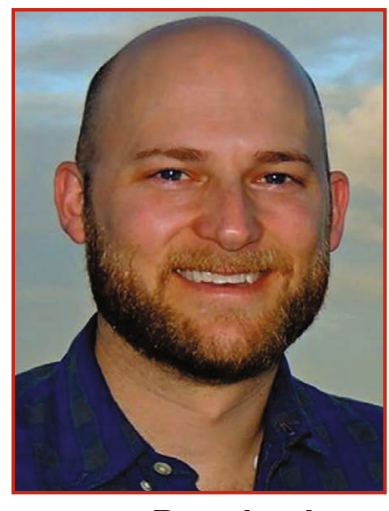

Brandon Lane 
microstructure, and mechanical behavior. Some of the key challenges to using a model-centric approach are: a) How can the software be validated to ensure that the code produces valid predictions? b) Can the various software tools be integrated? c) How do the uncertainties propagate through the different models?

AM-Bench will attempt to address these needs through a continuing series of controlled benchmark tests, in conjunction with a conference series, to allow modelers to test their simulations against rigorous, highly controlled additive manufacturing benchmark test data. The intent of the series is to create a single set of rigorous benchmark tests that developers can compare against, and to produce results that will benefit the entire AM community.

The first round of benchmark test challenges was made public in February 2018, covering laser powder bed fusion and individual bare-plate laser traces for metals, along with materials extrusion and selective laser sintering for polymers. The due date for blind simulation predictions is May 18. 2018. On May 19, all benchmark

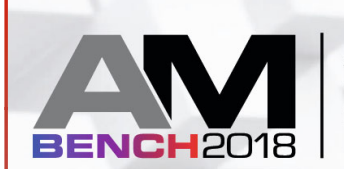

ADDITIVE MANUFACTURING BENCHMARKS

\section{Additive Manufacturing Benchmarks 2018 Important Dates}

\section{Symposium Abstract Deadline: March 1, 2018}

\section{Benchmark Tests Submission Deadline: \\ May 18, 2018}

Benchmark Measurement Results

Made Public:

May 19, 2018

Discount Registration Deadline: May 25, 2018

Housing Deadline:

May 25, 2018

For additional information and to register for this meeting, visit www.tms.org/ambench2018. measurement results

will be released,

and one month

later, the AM-Bench

2018 conference

will be held at the

main NIST campus

in Gaithersburg,

Maryland, from June

18-21. This conference will provide a forum where modelers and experimentalists can come together to discuss what works, what doesn't work, and most importantly, how we can fix what doesn't work. In the current scenario, a new series of benchmark tests with an associated meeting will take place every two years, with the conference moving between different international locations. Since TMS is recognized as the leading professional society for additively manufactured materials, the organizers of AM-Bench are delighted to partner with this organization so that we can open this important conference series to as wide an audience as possible. In addition to sessions specifically devoted to the benchmark tests and associated simulations, AM-Bench 2018 will also include a Measurement and Modelling Symposium that will examine quantitative measurements, state-of-theart models, end user modeling needs, and future modeling and measurement needs. Best practice presentations from internationally renowned experts will also be included.

In summary, AM-Bench 2018 is the first in a series of events focused on validating and improving the accuracy of model predictions and developing universally accepted quantitative measurement approaches for all AM materials and methods. Full details about the AM-Bench organization, benchmark tests, and timeline can be found on the AM-Bench website: www.nist.gov /ambench. Details about the AM-Bench 2018 conference can be found at www .tms.org/ambench2018. Note this series is open to the entire AM community regardless of whether or not one chooses to participate in the benchmark tests. All are welcome to join us!

\section{Lyle E. Levine is the project leader} for the NIST Materials Measurement Laboratory (MML) Additive Manufacturing of Metals Project, and serves as chair of the AM-Bench Steering Committee and co-chair of the AM-Bench Organizing Committee. Mark R. Stoudt, is a materials research engineer with the NIST Materials Measurement Laboratory and a member of the AM-Bench Organizing Committee. $\mathrm{He}$ is also vice chair of the TMS Materials Processing and Manufacturing Division (MPMD) Council and past chair of the TMS Shaping and Forming Committee. Brandon M. Lane is the project leader for the NIST Engineering Laboratory Realtime Monitoring and Controls for Additive Manufacturing Project and co-chair of the AMBench Organizing Committee.

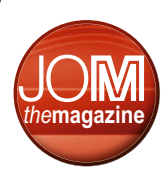

